\title{
Transorbital cerebellar airgun pellet injury
}

Sir,

Air guns, although considered to be toys, can cause injuries ranging from trivial to grievous. The potential of airguns for causing injury is underestimated. Recent concerns have been raised about the safety of them as toys ${ }^{[1]} \mathrm{A}$ rare case involving a young boy, who was accidentally hit by an air gun pellet during play, is described. Although there are many previous reports of intracranial air-gun pellet injuries, transorbital cerebellar injury due to air-gun pellets has not been reported previously in Medline.

We present a 15 -year-old boy was hit accidentally by an air gun pellet during play. There was a small enterance wound in the lower right orbital fold and he and his family were primarily concerned about his eyes on admission. Neurological examination including visual acuity and eye movements were intact. The Xray and CT sean showed the presence of an air-gun pellet to the posterior fossa [Figure 1a, b, c]. Other CT scan images showed that the air gun pellet travelled through the orbit without penetrating the globe. It passed into the middle cranial fossa and lodged in the posterior fossa.

Considering the trajectory of the pellet, the patient underwent cerebral angiography which was normal.

A suboccipital craniectomy in the prone position was performed and the air-gun pellet was removed as confirmed by a CT scan (Figure 1d). During the operation, we used only anteroposterior and lateral X-rays for localization of the pellet. Cefazolin and then oral cephalexin were adminstered to the patient for two weeks. No antiepileptics were administered to the patient. The patient had an uneventful postoperative course.

Air gun pellets are low velocity missiles that cannot pass easily through the adult skull bone. The possible points of entrance into the skull are through the eye, temporal and frontal bones and

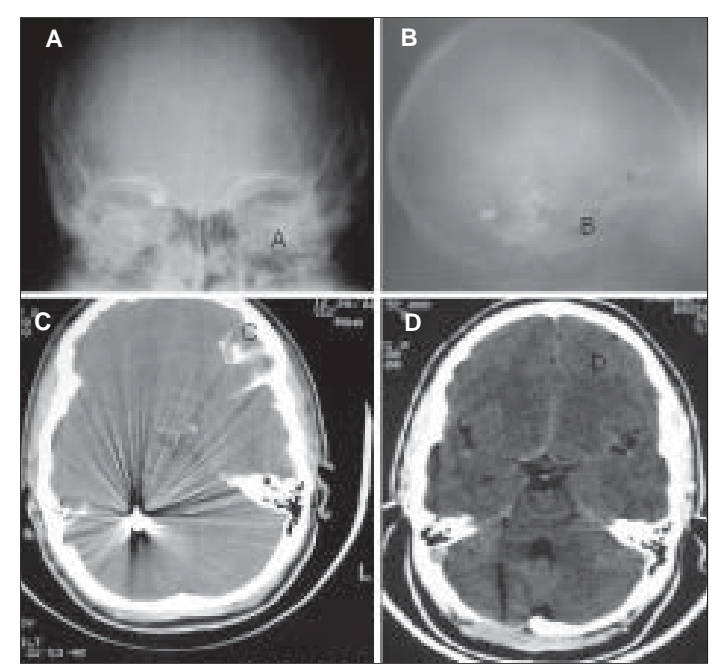

Figure 1: A. Anteroposterior skull X-ray illustrating the air gun pellet, B. Lateral skull view, C. Axial CT scan showing that the pellet has been lodged in the posterior fossa, D. Postoperative axial CT scan. 
nose. ${ }^{[1]}$ It is usually assumed that low velocity missiles in contrary to high velocity missiles ${ }^{[2]}$ do not penetrate the brain very far and lodge near the entry points ${ }^{[3]}$ In the present case the right eye has been the point of entrance. As in the majority of previous reports, the enterance wound was small. In one reported case the pellet entering through the orbit lodged in the occipital lobe. ${ }^{[3]}$ In other previous reports the pellet could not penetrate farther than the cavernous sinus when entering through the orbit, probably due to resistance in the trajectory of the pellet. ${ }^{[1,4]}$

Although the air-gun pellet could be removed rather easily in this case, use of ultraound and other intraoperative imaging modalities could be of great help in localization of the pellet in similar cases.

There is no evidence-based recommendation concerning antibiotics in such cases, but its administration seems rational. Since the air-gun pellet was lodged in the cerebellum, no antiepileptics were administered.

This case again shows the potential of air-guns for causing serious injuries. This and previous reports show that air-gun pellets can penetrate the brain far enough to injure any intracranial elements in their trajectory. ${ }^{[1,3,4]}$

Actions are needed to make airguns safer and reduce their availability to children and teenagers.

\section{Behzad Eftekhar, Mohammad Ghodsi, Ebrahim Ketabchi, Babak Esmaeeli}

Department of Neurosurgery, Sina Hospital, Tehran University, Iran.

E-mail: Eftekhar@sina.tums.ac.ir

\section{References}

1. Holland P, O'Brien DF, May PL. Should airguns be banned? Br J Neurosurg 2004;18:124-9.

2. Singh P. Missile injuries of the brain results of less aggressive surgery. Neurol India 2003;51:215-9

3. Amirjamshidi A, Abbassioun K, Roosbeh H. Air-gun pellet injuries to the head and neck. Surg Neurol 1997;47:331-8.

4. Gilmour DF, Ramaesh K, Fleck BW. Trans-orbital intra-cranial air gun injury. Eur J Ophthalmol 2003;13:320-3. 